Cahiers $d u$ MONDE RUSSE

\section{Cahiers du monde russe}

Russie - Empire russe - Union soviétique et États indépendants

$42 / 1 \mid 2001$

Varia

\title{
Le phénomène de la série culte en contexte
}

soviétique et post-soviétique.

L'exemple de Semnadcat' mgnovenij vesny

\section{Rodolphe Baudin}

\section{(2) OpenEdition}

Journals

Édition électronique

URL : https://journals.openedition.org/monderusse/8436

DOI : $10.4000 /$ monderusse. 8436

ISSN : $1777-5388$

Éditeur

Éditions de l'EHESS

Édition imprimée

Date de publication : 1 janvier 2001

Pagination : 49-70

ISBN : 2-7132-1388-6

ISSN : $1252-6576$

Référence électronique

Rodolphe Baudin, "Le phénomène de la série culte en contexte soviétique et post-soviétique. », Cahiers du monde russe [En ligne], 42/1 | 2001, mis en ligne le 01 janvier 2007, consulté le 03 septembre 2022. URL : http://journals.openedition.org/monderusse/8436 ; DOI : https://doi.org/ $10.4000 /$ monderusse. 8436 
chercher : repérer : avancer

Cet article est disponible en ligne à l'adresse :

http://www.cairn.info/article.php?ID REVUE=CMR\&ID NUMPUBLIE=CMR 421\&ID ARTICLE=CMR 4210049

Le phénomène de la série culte en contexte soviétique et post-soviétique. L'exemple de Semnadcat' mgnovenij vesny

par Rodolphe BAUDIN

| Editions de l'EHESS | Cahiers du monde russe

$2001 / 1$ - Vol 42

ISSN 1252-6576 | ISBN 2713213886 | pages 49 à 70

Pour citer cet article :

—BAUDIN R., Le phénomène de la série culte en contexte soviétique et post-soviétique. L'exemple de Semnadcat' mgnovenij vesny, Cahiers du monde russe 2001/1, Vol 42, p. 49-70.

Distribution électronique Cairn pour les Editions de l'EHESS.

(C) Editions de l'EHESS. Tous droits réservés pour tous pays.

La reproduction ou représentation de cet article, notamment par photocopie, n'est autorisée que dans les limites des conditions générales d'utilisation du site ou, le cas échéant, des conditions générales de la licence souscrite par votre établissement. Toute autre reproduction ou représentation, en tout ou partie, sous quelque forme et de quelque manière que ce soit, est interdite sauf accord préalable et écrit de l'éditeur, en dehors des cas prévus par la législation en vigueur en France. Il est précisé que son stockage dans une base de données est également interdit. 


\title{
LE PHÉNOMÈNE DE LA SÉRIE CULTE EN CONTEXTE SOVIÉTIQUE ET POST-SOVIÉTIQUE
}

\author{
L'exemple de Semnadcat' mgnovenij vesny
}

Nombreuses sont les études, tant en russe que dans les langues occidentales, consacrées au cinéma soviétique. La plupart d'entre elles portent sur le cinéma de l'avant-garde des années 20 (Vertov, Kulešov) ou sur l'œuvre d'Eisenstein. D'une valeur artistique incontestable - et c'est ce qui explique l'inflation du discours critique à leur propos - ces œuvres sont pourtant peu connues, sinon de nom, du grand public russe lui-même, à l'exception, peut-être, de celles d'Eisenstein. Beaucoup plus familières à ce public sont les œuvres appartenant à la culture de masse soviétique, et notamment à la culture télévisuelle. Le téléfilm soviétique, les séries produites à l'époque brejnevienne, et diffusées inlassablement sur le petit écran, sont très peu étudiées ${ }^{1}$, souvent du fait de leur faible qualité artistique, parfois aussi simplement parce qu'elles ne correspondent pas à l'horizon d'attente générique défini par des ouvres semblables - ou assimilables au niveau de leur mode de production ou de diffusion - en Occident.

L'une des plus célèbres séries soviétiques (et maintenant russes) des trente dernières années est sans doute la série Semnadcat' mgnovenij vesny (Dix-sept instantanés d'un printemps), réalisée en 1972-1973 par Tatiana Lioznova, sur un scénario, adapté de son roman, de Yulian Semenov.

La série raconte les aventures, au printemps 1945, lors de l'effondrement du III' Reich, de Stirlitz (Vjačeslav Tihonov), espion soviétique infiltré chez les SS, au

1. Ainsi signalons que l'ouvrage, dirigé par J.-L. Passek, Le cinéma russe et soviétique (Paris, L'Équerre, 1981, coll. « Cinéma Pluriel »/ Centre Georges Pompidou) ne fait pas référence à la série à laquelle je consacre cet article. 
sein du contre-espionnage allemand, où il occupe un poste important au « 4 bureau », sous les ordres de Schellenberg (Oleg Tabakov).

Chargé par Moscou de découvrir qui, au sein du haut commandement allemand, essaie d'entamer des pourparlers secrets de paix avec les Américains, Stirlitz doit également sauver la vie de Katia/Käte (Ekaterina Gradova), femme de son agent de liaison (tué lors d'un bombardement), qui a été arrêtée par la Gestapo, trouver un nouvel agent de liaison et établir, en passant par la Suisse, le contact avec Moscou. Enfin, Stirlitz est obligé de collaborer à l'établissement des contacts cités plus haut avec les Américains, puisqu'ils sont l'œuvre de Schellenberg et Himmler (Nikolaj Prokopovič), ses supérieurs directs, et doit échapper à la suspicion dont l'entourent les chefs de la Gestapo, Kaltenbrunner (Mihail Jarkovskij) et Müller (Leonid Bronevoj).

La série, diffusée immédiatement à la télévision, souvent deux fois par jour, l'après-midi et en deuxième partie de soirée, connut tout de suite un succès important, ce dont témoignent ses très nombreuses rediffusions ainsi que, dans un autre champ, les témoignages que j'ai pu récolter autour de moi auprès d'un public (urbain) âgé de 20 à 55 ans. Ainsi une connaissance de Saint-Pétersbourg d'une cinquantaine d'années m'a déclaré que, lors des premières diffusions, les Russes regardaient la série « avec passion » (zapoem). J'ajouterai que ce phénomène transcendait, et transcende encore, les catégories socioprofessionnelles puisque les enfants, âgés d'une vingtaine d'année, d'un chercheur en littérature de Moscou en parlent avec autant de chaleur que ceux d'un fonctionnaire du tribunal de Riazan ou d'un ingénieur d'Obninsk (région de Kaluga).

La popularité de cette série me semble pouvoir justifier qu'on lui consacre une étude, d'autant qu'une telle étude peut apporter des éléments pour déchiffrer une culture de masse soviétique trop méconnue en Occident, et qui, loin de s'effacer avec la disparition de l'URSS, est restée très présente.

Cette permanence de la culture de masse soviétique ne va pas, toutefois, sans transformations internes, notamment l'élévation de certaines de ses manifestations à la dignité de culture pop, phénomène de l'ordre de l'esthétisation, lié au passage de la Russie à la société de marché et à son ingestion des catégories culturelles du contexte post-moderne ${ }^{2}$.

Enfin, la popularité actuelle de cette série me semble être le signe, en même temps que l'instrument, d'une réappropriation par le public le plus jeune d'un passé culturel (sinon directement historique) commun, que l'invasion du champ des

2. La question de l'apparition de la problématique post-moderne en Russie soviétique puis post-soviétique a fait l'objet d'une très bonne étude par Leonid Heller : « Nécro-, rétro-, ou post? modernismes, modernité et réalisme socialiste », Cahiers du Monde russe et soviétique, 33(1), 1992, pp. 5-22. Dans son article, L. Heller semble inviter à multiplier les contributions dans ce champ de la recherche, encore peu étudié par les spécialistes francophones : «Depuis une dizaine d'années, la culture occidentale se félicite ou se désole de vivre une nouvelle époque, celle du 'postmodernisme'. Le terme est entré dans les mœurs et finit même par lasser un peu. Il garde en revanche toute sa nouveauté dans la discussion sur la culture russe. » (C'est moi qui souligne). 
médias russes par les produits occidentaux, depuis le début de l'ère Eltsine, a relégué dans l'ombre ${ }^{3}$.

Je me propose, dans un premier temps, d'étudier l'esthétique de cette série télévisée et de la comparer avec celle des séries cultes occidentales, comparaison qui s'est imposée à mon esprit lors de la première vision, et qui doit s'imposer aux plus jeunes téléspectateurs russes, abreuvés depuis le début des années 90 de séries américaines, ou au public plus âgé - entre 20 et 30 ans - qui a suivi la série une première fois dans les années 80 et la redécouvre maintenant, alors que son œil est habitué au traitement américain de la violence et de l'action, tel qu'il est présenté par les séries citées plus haut.

Dans un deuxième temps, $\mathrm{j}$ ' analyserai les conditions et les rituels de la réception de la série par le public, ce qui me mènera à me demander ce que peut être le culte de la série télévisée (la série culte, pour employer le syntagme courant depuis quelques années) en contexte soviétique et post-soviétique, le phénomène me paraissant caractéristique avant tout de la culture de la société de marché, culture encore inexistante, sinon dans ses formes les plus grossières (comme la «starisation » des chanteurs de variété, mise en scène par la presse people russe récemment apparue) en Russie (il me semble ainsi qu'une chaîne comme la chaîne câblée « série-club » ne pourrait pas encore exister en Russie) $)^{4}$.

Dans une étude sur la réception et la temporalité, Violette Morin ${ }^{5}$ distingue le feuilleton de la série. Le premier est défini par la présence entre ses différents segments de «liens de progression irréversible qui conditionnent la suite [des] épisodes »; la seconde par un lien de « coexistence » s'appuyant sur la récurrence de mêmes personnages ( « héros séduisants ou sympathiques »), ou sur celle d'un même « climat référentiel » comme le Far West ou la science-fiction (deuxième définition un peu large, que l'on pourrait réduire à celle d'un même univers de référence, non

3. Sur la popularité toujours actuelle de la série, cf. A. Filipov, « A Vas, Štirlic, ja poprošu ostat'sja... » (Quand à vous, Stirlitz, je vous prierai de rester...), Izvestija, 23 juillet 1999 (sur le $75^{\mathrm{e}}$ anniversaire de la réalisatrice Tatiana Lioznova), p. $7:$ : Et pourtant ses films ne vieillissent pas. Ils sont regardés et par les vieux et par les jeunes, et les plaisanteries consacrées à Stirlitz, contrairement à celles sur Brežnev, ne sont pas près de disparaître. Visiblement, le faux Standartenführer vivra éternellement. » (C'est moi qui souligne).

4. Le problème de l'existence d'un culte pour les séries télévisées en Russie n'a été, à ma connaissance, que peu étudié. On pourra se reporter néanmoins à un article d'inspiration sociologique consacré aux pratiques d'audience du public russe des années 1990 face aux soap operas : cf. V. P. Dubickaja, « Teleserialy na ekrane i v postsovetskoj mifologii » (Les séries télévisées dans la mythologie post-soviétique), Sociologičeskie issledovanija, 9, 1996, pp. 77-82. Les séries retenues pour l'analyse dans cet article sont majoritairement des productions brésiliennes. Deux séries russes (soviétiques) y sont toutefois prises en compte : «Les seules séries soviétiques (Večnyj zov, Teni isčezajut v polden' (L'éternel appel, Les ombres disparaissent à midi)) attirent un nombre significatif d'hommes et de téléspectateurs des groupes d'adultes actifs. » (p. 79). Notons ici que ces deux productions sont des séries, alors que Semnadcat' mgnovenij vesny, comme je l'explique plus loin, est plutôt un serial. On consultera encore, sur le phénomène de l'apparition de films cultes en Russie, et plus particulièrement à Moscou, l'article de Sergej Kuznecov, «Kul'tovoe kino na zakate » (Le déclin du cinéma culte), Hudožestvennyj žurnal, 18, 1997, pp. 40-41. On y trouvera notamment une analyse comparée des termes « kul' tovyj » (culte), « modnyj » (à la mode) et « populjarnyj » (populaire) (p. 41).

5. Cf. V. Morin, « Le présent actif dans le feuilleton télévisé », Communications, 39, 1984,p. 240. 
pour les spectateurs, comme univers lisible, comme code, mais pour les personnages, comme univers « réel », c'est-à-dire comme réserve de possibles narratifs).

Si l'on s'en tient à cette définition, Semnadcat' mgnovenij vesny est un feuilleton (ou serial) à fin unique, fin dans laquelle chaque ligne narrative trouve son épuisement ou sa résolution.

La série se compose de douze épisodes de 70 minutes. Comme l'exige la poétique du feuilleton, chaque épisode se termine brusquement, à un «moment crucial » (le fameux cliffhanger), incitant ainsi le spectateur à regarder l'épisode suivant, afin de découvrir comment va se développer ou se résoudre la situation amorcée à la fin de l'épisode précédent. Cette mécanique alterne deux modèles structuraux essentiels : soit le personnage en sait plus que le spectateur (info personnage $>$ info spectateur), ce qui suscite chez ce dernier le désir d'apprendre ce que le personnage, lui, sait déjà, soit le spectateur en sait plus que le personnage (info personnage < info spectateur), et se demande comment le personnage va réagir quand il va apprendre ce que lui a déjà appris.

La fin du 7 e épisode illustre la première variante : Stirlitz rentre chez lui et y découvre qu'un homme l'attend. Grâce à l'utilisation du champ sans contrechamp, le spectateur comprend que Stirlitz voit l'homme et le reconnaît, sa surprise et sa crainte apparaissant de surcroît sur son visage, mais comme le contrechamp attendu n'arrive pas, le spectateur est obligé d'attendre le 8 e épisode pour découvrir qui est ce mystérieux visiteur.

La fin du 8 e épisode illustre l'autre variante. Stirlitz est presque démasqué. La Gestapo le fait rechercher pour l'interroger. Une série de plans montre manœuvres de soldats et formation de barrages routiers. Mais Stirlitz ne sait pas qu'il est recherché. La fin de l'épisode le montre arrêté dans sa voiture au bord de la route, décidé à faire un somme d'une demi-heure afin de ne pas risquer de s'endormir au volant. Le spectateur imagine donc qu'il va repartir au début du 9e épisode, et se demande comment il va franchir ces barrages (ce que l'épisode suivant ne montre d'ailleurs pas, pour des raisons que nous analyserons plus bas).

La structure interne de la série est très stable. Comme pour les clausules, la composition même des épisodes est assez répétitive (ce qui logiquement, est plus caractéristique de la série que du feuilleton, puisque la série, pour assurer la cohérence de ses personnages - par reconnaissance de leurs traits distinctifs - est obligée de les replacer encore et toujours dans des situations similaires).

Cette répétitivité est d'abord celle du découpage. Chaque épisode est divisé en journées, chaque journée en heures. Ce découpage est aléatoire : il divise l'épisode en tranches inégales dont le début est identifié par un timing intérieur à la fiction très précis. En réalité, en plus de son rôle d'agent du découpage et de modulateur rythmique (ce que souligne le fond sonore, reproduisant le tic-tac d'une horloge), ce timing, réalisé par des intertitres apparaissant en volets, a une fonction pragmatique. Il souligne dans l'esprit du spectateur la précipitation du feuilleton vers sa fin ${ }^{6}$.

6. Enfin, ce timing en intertitres concourt à créer un effet de réel, soulignant l'inscription du récit dans l'Histoire, et renvoie au temps irréversible du mythe. 
Au niveau de la composition de l'ensemble, chaque épisode alterne trois types fixes de séquences définies par leur nature et leur fonction :

- séquences extranarratives-discursives, statiques (et très théâtrales), où sont développés les grands thèmes du feuilleton (nature du bien et du mal, libre arbitre, salut de l'homme - faut-il sauver les Allemands d'eux-mêmes?), mises en récit par de longs dialogues;

- séquences narratives, montrant essentiellement des déplacements (à pied dans les couloirs de la Kommandantur, en voiture ou en train entre deux lieux de Berlin, entre deux pays) et permettant de relier les séquences du premier type;

- séquences mixtes (narratives et discursives) enfin, composées d'images d'archives montrant les combats de l'avancée des Alliés (essentiellement soviétiques) vers Berlin, quelques événements marquants de l'histoire de l'Allemagne d'avant le fascisme, ou de l'histoire de l'URSS.

L'utilisation de ces séquences d'archives peut être justifiée pleinement au regard de la diégèse. Dans ce cas, ces images sont présentées à l'intérieur même de la fiction, comme images d'archives. Elles ont le même statut logique dans l'univers de la fiction (la diégèse) que dans la réalité : dans les deux cas, elles sont des objets. Ainsi leur première utilisation, dans le premier épisode, est-elle justifiée de cette manière, qui montre Himmler et Schellenberg, personnages ici fictionnels, regardant les actualités des Alliés, objet non fictionnel, mais intégré à la diégèse.

Plus fréquemment, leur utilisation est motivée narrativement mais n'est pas justifiée logiquement. Ainsi lorsque elles servent de flash-backs insérés après un gros plan montrant Stirlitz se plongeant dans ses pensées (souvenirs « personnels » du héros sur l'Allemagne - ouvrière et pro-bolchévique ici - de la République de Weimar, sur Paris avant guerre, de Gaulle, ou Moscou en liesse après la victoire de Stalingrad). Dans ce cas, elles n'ont plus le statut d'objets appartenant à l'univers de la fiction, mais sont intégrées au matériau narratif. D'objet de l'histoire, elles deviennent véhicule du récit. D'événements regardés par les personnages, comme plus haut, elles deviennent événements vécus par ces mêmes personnages. Leur présence est justifiée par le fil du récit, puisqu'elles mettent en image les souvenirs de Stirlitz, mais est illogique au regard de la cohérence interne de la diégèse, et donc de la règle d'illusion référentielle, ne serait-ce que parce que leur grain, différent de celui de la pellicule employée pour filmer la partie fictionnelle, trahit leur statut de remplor?.

Le plus souvent enfin, elles ne sont pas justifiées autrement que par le découpage de l'action, qu'elles participent à créer en même temps qu'elles le justifient.

Séquences discursives alternent donc avec images d'archives, les deux types étant reliés par cette «colle fictionnelle » légitimante que sont les séquences narra-

7. Les occurrences citées plus haut - images de Stalingrad ou de De Gaulle — sont parmi les nombreux exemples de « trou » dans l'illusion référentielle, causés par l'utilisation, au sein du matériau fictionnel, des images d'archives. En effet, il est répété plusieurs fois dans le feuilleton que Stirlitz est en Allemagne depuis vingt ans (c'est-à-dire depuis 1925), aussi comment aurait-il pu assister à la célébration de la victoire de Stalingrad à Moscou, ou pu voir de Gaulle en exil en Angleterre? Il y a ici conflit - mal géré, et c'est un des défauts majeurs du feuilleton - entre la fonction didactique et la fonction de fiction distrayante. 
tives, presque réduites à leur fonction de liaison, tant leur durée est diminuée face à celle des longs dialogues psychologico-thématiques. Car c'est là la première différence essentielle séparant Semnadcat' mgnovenij vesny des feuilletons télévisés français ou américains à thématique militaire ou d'espionnage. Le discours y est hypertrophié au détriment des péripéties, et surtout, au détriment de l'élément essentiel du feuilleton américain — modèle du genre,- l'action.

L'action est effectivement pratiquement absente de cette série culte soviétique. De même qu'y est bannie la représentation d'un conflit ou d'une violence autre que psychologique ${ }^{8}$. Les seules scènes d'action ou scènes montrant l'extériorisation d'une lutte sont celle de l'élimination par Stirlitz d'un agent allemand, Klaus, à la fin du 2e épisode, filmée avec une discrétion étonnante (champ/contrechamp, séparant le coup de feu de son résultat, la mort de l'homme), celle de l'interrogatoire de Käte par la Gestapo à la fin du 9e épisode et celle de la filature en voiture, filature et non poursuite ( $5^{\mathrm{e}}$ épisode).

L'unique scène représentant la violence d'une manière directe - scène de torture dans les caves de la Gestapo - est étrangement non réaliste (plastiquement) et touche au fantasmatique (homme pendu par les pieds et SS avec des fouets), au naïf populaire (gravures représentant l'enfer), au littéraire archaïque distancié.

Tout le reste (violence, mais aussi, plus simplement, action) est « mangé » par le discursif, le paradigmatique, la déclinaison dans la fiction des thèmes de l'histoire. Au point que la seule action représentée, dans le sens de représentation d'une violence, d'un conflit, semble être assumée par les images d'archives montrant combats et explosions de la Deuxième Guerre mondiale (et encore la nature historique, réelle, du matériau, tend à mettre cette action à distance, en la renvoyant dans le domaine de l'épique, du mythique, phénomène paradoxal à première vue dont nous reparlerons plus loin). L'action est ainsi reléguée au second plan (et au mythe), mais aussi à l'effet de réel historique (replacer l'énoncé dans un contexte en communication avec le réel). À l'inverse, et de manière complémentaire, l'effet de réel fictionnel est hissé au rang d'action. En effet, si Stirlitz ne se bagarre pas, ne tire pas sur ses adversaires, on le voit en revanche jardiner, cuisiner, ou, détail étonnant et répété d'un épisode à l'autre, ranger sa voiture dans la cour de sa villa. Cette hypertrophie du légitimant au niveau thématique (un espion doit être discret, mener une vie ordinaire pour ne pas attirer l'attention, aussi le feuilleton décline les situations types de la vie de l'homme ordinaire, au détriment de l'intérêt dramatique) met l'accent sur l'intériorité, sur l'horizontal, sur la psychologie et le thématique.

Contrairement à ce que l'on trouve dans les James Bond, où « tant le personnage que l'auteur résolvent le problème [du droit de donner la mort à l'autre] autrement que par voie thérapeutique, c'est-à-dire en excluant la névrose de l'univers des possibilités narratives ${ }^{9}$, dans Semnadcat' mgnovenij vesny, tous les possibles

8. La violence et le conflit sensibles tout au long de la fiction sont ceux qui opposent les SS entre eux, entre membres d'administrations différentes. Le conflit est ainsi conflit larvé entre fonctionnaires, ce qui est peut-être très révélateur de l'Union Soviétique des années 1970 qui a produit le feuilleton.

9. U. Eco, « James Bond : une combinatoire narrative », Communications, 8, 1966 ; id., L'analyse structurale du récit, Paris, Seuil, 1981, p. 83. 
narratifs et filmiques sont au service du psychologique. C'est ce choix qui rejette l'action hors du récit. Le «tout psychologique » se traduit dans le filmage par le nombre important des gros plans sur le visage de Stirlitz (on notera à ce propos l'importance donnée au travail de l'acteur, conformément à une tradition très russosoviétique) qui éjectent dans le hors-champ le monde fictionnel comme théâtre d'aventures possibles. L'espace - horizontal - du conflit entre les corps, entre les objets surtout, n'est pas pris par la caméra, toujours au plus près des visages, obligeant ainsi l'action à se résorber dans le vertical, dans la psychologie. On notera à ce propos que les scènes d'extérieur sont rares, et que les scènes d'intérieur réitèrent un nombre réduit de lieux (le café «Elefant », la villa du héros, son bureau) comme dans le soap opera psychologique occidental.

La série essaie ainsi, dans la tradition psychologique, de construire un «vrai » personnage, et rejette le personnage-fonction du feuilleton d'action occidental, dont la présence physique à l'écran n'est que la légitimation aux niveaux narratif et thématique d'un montage produisant un énoncé visuel à consommer, déroulant des éléments définis préalablement par un horizon d'attente draconien : courses de voitures, fusillades, bagarres, chutes, explosions.

Le héros ici, ne livre pas un énoncé prédéterminé, mais se livre, non dans l'événementiel, le syntagmatique, (puisqu'il est espion!), mais dans le thématique. Le héros voudrait ne pas être le « fil gris ${ }^{10}$ du récit, il est néanmoins celui du thématique.

Le thématique construit le «portrait» du personnage, que la fiction se contente de brosser superficiellement. En le plongeant dans des situations critiques, le récit se contente, car il fait toujours sortir le héros de ces situations, de souligner son statut actanciel, mais ne l'individualise pas. Seules les séquences thématiques, anarratives (dans le sens où elles sont amovibles dans l'économie du récit) informent sur le personnage. À ce titre, il est intéressant de comparer la séquence du jeu dans Semnadcat' mgnovenij vesny et dans la série des James Bond ${ }^{11}$.

Dans chaque épisode de la série adaptée de Ian Flemming, Bond se retrouve devant une table de jeu dans un casino. Ces séquences ont deux fonctions : caractériser le héros (le jeu connote le goût du risque, le sang-froid et l'habileté, sinon l'intelligence) et faire avancer le récit (la séquence est toujours le lieu d'une première rencontre et d'un premier duel, métaphorique, « joué » avec l'adversaire

10. Cf. V. Chklovskij, «La construction de la nouvelle et du roman », in Théorie de la littérature, textes des formalistes russes réunis, présentés et traduits par Tzvetan Todorov, Paris, Seuil, 1966, p. 190

11. La comparaison entre Stirlitz et Bond, si elle paraît oiseuse (types d'énoncés filmiques différents, modes de diffusion dissemblables, et, au niveau diégétique, cadres spatio-temporels différents), est imposée par les Russes eux-mêmes. Cf. l'article des Izvestia déjà cité : « Et le Standartenführer Stirlitz, création immortelle de Tatiana Lioznova, n'est pas pour rien si différent de son collègue anglo-saxon James Bond. Pour l'Anglais, c'est le résultat qui comptait, pour le Russe, le processus; chez Maksim Maksimovič Isaev, la réflexion prédominait sur l'action. Pour cette raison, il est amené à durer. » Cette comparaison me semble être le signe de la (ré)inscription de Semnadcat' mgnovenij vesny dans le paradigme, nouveau pour lui, de la culture russe occidentalisée (américanisée) de l'ère eltsinienne. 
ou son substitut, la femme fatale). Notre feuilleton soviétique utilise le même type de séquence (en réponse, peut-être, à la série anglo-américaine, ou parce que la thématique des deux séries est la même) : Stirlitz joue au café « Elefant », lieu dans lequel il se rend dans chaque épisode (ou presque), mais n'y rencontre personne qu'il ne connaisse déjà, et n'y reçoit donc aucune information nouvelle. Ici, la séquence a purement pour fonction de définir le caractère. Il est amusant de noter au passage les équivalences, d'une mythologie à l'autre : les deux espions jouent contre un adversaire féminin, mais alors que Bond joue à un jeu de hasard - pour de l'argent de surcroît - contre une femme, pur objet érotique, Stirlitz joue aux échecs (jeu qui connote l'intelligence du héros - si Bond est l'homme de l'action, son homologue soviétique est celui de la réflexion - et jeu non mercantile), contre une vieille dame inoffensive et désexualisée ${ }^{12}$.

Ce type de séquences n'a pas d'autre but que de caractériser Stirlitz comme étant uniformément bon, intelligent, bienveillant, voire débonnaire (il perd son temps à jouer aux échecs avec une vieille dame), c'est-à-dire parfaitement monolithique, plus encore que Bond, chez qui s'affrontent le Devoir et le Désir (même si cela est traité d'une manière sommaire).

Pas d'action donc, mais seulement de la psychologie, et encore, une psychologie primaire (pas de désir, comme chez Bond, mais le seul Devoir), s'échappant dans le thématique, afin de ne pas présenter de personnages conflictuels. Cette absence de conflit chez Stirlitz confirme son statut de héros positif (položitel'nyj geroj), et explique cette réduction de la psychologie au thématique et à l'idéologique. Stirlitz a une psychologie, mais une psychologie apaisée, dont les mouvements recoupent parfaitement le discours monolithique de l'idéologie, que le comportement idéal du héros positif véhicule. Cette psychologie n'exclut pas le doute, mais ce doute n'est pas une remise en cause de l'idéologie. À l'inverse, il exprime l'angoisse du héros à l'idée de ne pas être à la hauteur de sa fonction d'exemple idéal ${ }^{13}$.

Le rattachement de Stirlitz au type du « héros positif » intègre la série dans le système esthétique du cinéma soviétique, et permet de contrebalancer le trouble dangereux - car sûrement agréable - provoqué probablement chez les spectateurs par cette étrange et contradictoire figure de héros soviétique («naš») en uniforme de SS ( «ne naš »).

Les aspects les plus ridicules du héros positif, qui rappellent les clichés d'un cinéma soviétique plus ancien et idéologiquement plus naï - Stirlitz est asexué, et

12. Épisode 8. Notons à ce propos que Stirlitz joue avec les pions blancs, ce qui le connote comme personnage positif. Lui faire prendre les noirs aurait illustré l'ambiguïté du personnage, et introduit un flottement du sens risqué pour un feuilleton à si forte charge didactique, destiné de plus à un public (plutôt) jeune.

13. Sur le problème du doute du héros positif, on consultera A. Pankov, « Vosled geroju. Problema položitel'nogo geroja v sovremennoj kritike » (Sur les traces du héros. Le problème du héros positif dans la critique contemporaine), Voprosy literatury, 5, 1984, pp. 3-30. Plus généralement, le héros positif a fait l'objet d'une bonne synthèse par R. W. Mathewson, The positive hero in Russian literature, 2e ed., Stanford, Stanford University Press, 1975. 
sacrifie sa vie tout entière à sa mission ${ }^{14}$ - expliquent en partie, comme je le montrerai plus loin, le nombre considérable d'histoires drôles que le personnage suscite.

En même temps qu'il actualise certains traits du «héros positif » archaïque, Stirlitz en réalise d'autres, rapportables à une variante plus moderne du type. Le «héros positif» ne peut pas en effet ne pas épouser les évolutions de la société soviétique. À ce titre, il n'est pas surprenant de constater chez Stirlitz un certain goût pour le mode de vie bourgeois, goût répandu dans les classes moyennes et supérieures de la société soviétique des années 1970 (Stirlitz est un esthète mais aussi un gourmet : il boit du cognac français, régale ses deux compagnes du café «Elefant » - la vieille dame et la jeune dactylo - d'un dîner fin préparé par ses soins) $)^{15}$.

L'hypertrophie du thématique notée à l'instant est liée à l'aspect très littéraire du feuilleton. De même, cette forte influence du littéraire se sent dans le filmage. Ainsi le montage est presque absent de Semnadcat' mgnovenij vesny ou il est très rudimentaire. Une autre «grossièreté » du filmage liée à l'influence du littéraire me semble être l'utilisation constante du zoom pour recadrer les visages (le psychologique). Grossièreté courante dans le soap opera occidental, elle est ici de surcroît le signe d'une certaine approche du matériau fictionnel, comme par l'extérieur (presque comme si le texte lui-même était filmé), au lieu d'une approche par l'intérieur, qui passerait par un découpage filmique et un montage.

Cette importance du littéraire apparaît également dans l'utilisation de la voix off, qui introduit, à la place de la caméra, un narrateur assurant une fonction informative (sur les pensées de Stirlitz) et une fonction de régie («que va-t-il faire maintenant? ») exactement comme dans un énoncé romanesque ${ }^{16}$.

Absence d'action, camouflage de la violence - sauf dans le domaine, très abstrait, du mythique, où elle devient pure catégorie morale - inflation du discours, porté par des personnages dont c'est l'unique fonction, tous ces aspects semblent situer ce feuilleton soviétique bien loin des séries américaines à la théma-

14. Stirlitz est en effet seul, séparé de sa femme depuis vingt ans, et met à distance le lien érotique possible avec Gaby (Svetlana Svetličnaja), la jeune habituée de l' «Elefant », ou avec Käte, ce qui le désexualise. Apologie du refoulement au nom de la raison d'État, c'est là un trait évident du discours totalitaire. En fait il semble n'entretenir de rapport intime qu'avec Shellenberg, son supérieur hiérarchique (de nombreux plans les montrent, complices, riant dans les coins et se moquant de leurs collègues lors de réceptions officielles). Mais ceci n'est probablement pas suffisant pour y voir une marque d'homosexualité latente. Le rapport entre les deux hommes est plutôt de l'ordre du rapport père/fils, avec un fils - Stirlitz - qui trahira la confiance du père et s'en ira.

15. Cf. A. Amalrik, L’Union soviétique survivra-t-elle en 1984?, Paris, Fayard, 1970, p. 112, note 27 : «Nous observons aujourd'hui une aspiration croissante à la vie tranquille et au confort, voire une sorte de culte du confort dans toutes les couches de la société, surtout dans ses couches supérieures et moyennes. »

16. Ajoutons que la présence de cette voix off a encore pour fonction de créer de l'action, puisqu'elle introduit un conflit entre ce que fait Stirlitz et ce qu'il pense. Il y a ici réduction de l'action (filmique) à l'intérêt dramatique (littéraire). 
tique comparable ${ }^{17}$. Ceci s'explique par la double nature d'énoncé didactique et de métalangage idéologique de la série. Si les séries américaines sont des discours très chargés idéologiquement (elles confirment l'ordre des choses, la hiérarchie : domination de la femme par l'homme, de l'individu par la collectivité), elles revendiquent rarement ouvertement leur fonction didactique et leur statut de langage pour déchiffrer le réel (même une série comme Star Trek, avec son discours articulé et sa très forte transparence idéologique).

Car Semnadcat' mgnovenij vesny est avant tout un discours fictionnel sur le réel présent, et une encyclopédie pour interpréter ce réel.

Fonction encyclopédique d'abord: l'utilisation d'un nombre important d'images d'archives - même si leur déroulement au milieu de l'intrigue tend à les fictionnaliser - souligne la fonction didactique du feuilleton. Ces images sont souvent accompagnées de commentaires descriptifs et explicatifs, qui voudraient informer le (jeune) spectateur, même si ces archives, plutôt qu'informer, semblent décliner un discours fixe condamné à être repris et transmis comme énoncé à répéter, à l'instar du récit mythique. Plus évidentes sont ces séquences appelées « information pour la réflexion » («informacija $k$ razmyšleniju ») et introduites par une incrustation de ce titre sur l'écran. Presque toujours, ces séquences sont des notices historio-biographiques (sur Göring, Bormann, Himmler ou Goebbels) émaillées de citations historiques (récitées par la voix off) choisies pour leur barbarie. Elles sont introduites dans le récit comme extériorisation (pendant de la voix off) des réflexions de Stirlitz sur ces mêmes personnages. Mais la présence de l'incrustation est le signe de l'instauration d'une communication supplémentaire avec le téléspectateur. S'il est demandé à Stirlitz de déduire de ce portrait historique si tel ou tel dignitaire du Reich aurait intérêt à contacter secrètement les Américains, il est demandé au spectateur non une déduction « policière » du même ordre, mais une mémorisation du récit des atrocités commises par les nazis et de leurs déclarations abjectes (sur les juifs, la race allemande, la gloire des armes), mémorisation à faire suivre de leur condamnation absolue.

Cette espèce d'interactivité (télé-guidée, certes) du feuilleton, est la marque de l'importance de sa fonction didactique, de son rapport direct à l'encyclopédie.

Un autre phénomène, proche du premier, est lié à l'utilisation de l'image d'archive comme flash mémoriel (comme souvenir) de Stirlitz. Dans le 4e épisode, Stirlitz se rappelle Ernst Thälmann, héros (martyr, conformément à la mythologie soviétique) du mouvement ouvrier allemand des années 1920. Ce qui, dans la fiction, est censé être l'évocation par le héros d'un de ses amis (ce qui tend à le faire entrer dans l'univers de référence de la fiction, dans l'ensemble de ses possibles, et dans un deuxième temps, contribue à mythifier le réel historique) se transforme en

17. D'autres feuilletons soviétiques s'en rapprochent en effet beaucoup plus, ainsi l'autre série culte, à thématique policière celle-ci, Mesto vstreči izmenit' nel'zja (On ne change pas le lieu d'un rendez-vous), réalisée en 1979 par Stanislav Govoruhin, avec Vladimir Vysotskij et Vladimir Konkin dans les rôles principaux. Signalons par ailleurs que cette deuxième série est postérieure à Semnadcat' mgnovenij vesny et ne compte que cinq épisodes, de 70 minutes également. Il serait enfin intéressant de comparer les 70 minutes des épisodes des feuilletons russes aux inévitables 50 minutes des feuilletons américains. 
notice biographique détaillée avec chronologie, au mépris des règles de l'illusion référentielle.

Ce deuxième type de séquence rappelle un peu le fonctionnement d'un CDRom, mais inversé. Alors que dans un CD-Rom la sélection de l'information précède son « déroulement » (explication + illustration) - souvent une séquence animée ou filmée - ici, la séquence filmée (animée) de la fiction, s'interrompt pour introduire une séquence informative, mal intégrée (le procédé est peu discret) dans l'énoncé fictionnel.

La série est également discours idéologique et instrument d'interprétation du réel.

Le centre de l'intrigue de Semnadcat' mgnovenij vesny est occupé par la tentative de Himmler et Schellenberg d'entamer des pourparlers secrets avec les autorités américaines, pour obtenir une paix séparée et un soutien contre les soviétiques (la monnaie d'échange de l'émissaire allemand, le général Wolf, est la haine commune des nazis et des Américains pour les bolcheviks, haine soulignée à de nombreuses reprises dans l'histoire). Dans les échanges entre Stirlitz et Moscou, ces pourparlers sont désignés comme pourparlers «avec l'Ouest» («s Zapadom ») ; l'insistance avec laquelle est répétée cette expression, associée dans l'esprit des spectateurs des années 1970 au discours politique et idéologique contemporain, souligne l'anachronisme lexical : «Zapad » est, en 1970, le camp de l'Europe de l'Ouest et des États-Unis - formé après la fin du conflit mondial caractéristique du contexte de la guerre froide ${ }^{18}$. Dans ce contexte, les Américains sont marqués très défavorablement. Ce marquage négatif est légitimé rétroactivement par le fait qu'ils ont accepté de traiter avec des nazis, SS de surcroît (responsables directs, pour ce qui est de Himmler, de la «solution finale», dont le feuilleton donne à voir des images).

Le feuilleton donne donc des éléments d'appréciation des Américains.

L'appréciation réservée aux Allemands ressortit au même type de fonctionnement. Afin toutefois de ne pas assimiler uniformément les Allemands aux nazis (car les Allemands sont, partiellement, des alliés, les alliés de la RDA), le feuilleton emploie, face aux «mauvais » Allemands, de «bons » Allemands. Ces «bons » Allemands sont, par exemple, le professeur Pleschner, joué par Evgenij Estigneev, intellectuel lettré spécialiste de livres anciens (et le sème « ancien » est d'importance car il connote toute la diachronie de la culture allemande). Le feuilleton multiplie de plus les déclarations d'amour à l'Allemagne de la part de Stirlitz. Ainsi les meilleurs parmi les Allemands (les intellectuels, les gens simples et bons, comme la vieille dame avec qui Stirlitz joue aux échecs) travaillent avec les soviétiques, et les bouchers (les nazis) avec les Américains. Le feuilleton, fiction située dans un passé qu'il contribue à rendre mythique, entérine ainsi l'état des choses actuel et incite le public soviétique des années 1970 à confirmer la partition RFA $(=\mathrm{USA}=$ nazis $) / \mathrm{RDA}(=\mathrm{URSS}=$ fleur de la culture allemande $)$.

18. Dans le langage écrit, ce glissement joue sur le choix de la majuscule, ou non. Si l'on écrit zapad, c'est à la zone géographique que l'on fait référence, si l'on écrit Zapad, c'est au camp politique. Cf. la définition de ce terme dans le dictionnaire d'Ožegov. 
Résumant et donnant à interpréter et évaluer (dans un seul sens possible) le contexte de la guerre froide, le feuilleton fait également un sort à la France. Conduisant le pasteur Schlag (Rostislav Pliatt) à la frontière suisse (8e épisode), Stirlitz écoute (de manière anachronique) Piaf à la radio. Alors que le pasteur voit dans les deux chansons enchaînées («Milord» et «Non, rien de rien... ») le signe de la décadence de l'époque, Stirlitz, en Russe bon teint (soviétisé) apprécie la France, et se rappelle Paris et de Gaulle (séquence de souvenirs présentant un montage d'images d'archives, encore une fois non légitimée fictionnellement puisque l'on se demande où Stirlitz, bloqué en Allemagne depuis 1925, a pu voir le général). Ainsi la France est marquée moins négativement que les États-Unis, mais est réduite à un espace culturel (et non politique), résumé de tous les clichés de l'imaginaire russe puis soviétique sur la culture française. Le peu d'importance accordé à l'anachronisme (les chansons de Piaf datent des années 1950) souligne cette réduction de la France à un mythe culturel.

Mais si le didactique et l'idéologique semblent fonctionner de concert (l'idéologique régule le didactique, qui transmet l'idéologique), ils peuvent entrer en conflit, si le didactique donne à voir un matériau pouvant échapper à sa régulation, comme les images d'archives citées plus haut. L'effet de réel peut basculer dans le réel sans effet, les images d'archives «parler» toutes seules, cesser d'être la représentation contrôlée d'un monde à la signification déjà fixée, pour devenir matière à générer un discours critique. Aussi le feuilleton travaille avec une troisième catégorie, régulant à son tour les rapports entre réel et fictionnel, le mythe, ou plutôt la fabrication du mythique.

Le travail sur le mythe mené par Semnadcat' mgnovenij vesny passe d'abord par le brouillage des limites entre réel et fiction, brouillage opéré dans les deux sens.

Le réel des images d'archives tend à rendre la fiction véridique, afin de l'aider à assurer sa fonction didactique, et lui fournir sa légitimité comme énoncé à consommer. Notons à ce propos que l'absence de cette forme de légitimation dans une société de type capitaliste - dans laquelle le problème est résolu par l'argument « plaire au public » - se traduit dans les feuilletons télévisés occidentaux par une surfictionnalisation de leur contenu. Ainsi, très vite, certaines séries ou feuilletons télévisés ressortissant à la catégorie « policier » ont investi les sphères thématiques de la science-fiction ou du fantastique (le fantastique gangrena rapidement une série comme Chapeau melon et bottes de cuir par exemple), et ceci au mépris $\mathrm{du}$ respect du vraisemblable, le feuilleton semblant apporter avec lui ses propres codes de déchiffrement, décidant lui-même de ce qui peut, ou ne peut pas, être accepté par son public, et clamant son statut de pure fiction divertissante.

À l'inverse, notre série soviétique tente de gommer son identité fictionnelle en ayant recours à l'Histoire ${ }^{19}$. Mais en même temps le récit fictionnalise ces images d'archives (transformées en séquences d'action), pour contrôler la réception du

19. La méfiance vis-à-vis du fait fictionnel et le rejet de ses formes les plus ouvertement métadiscursives semblent accompagner depuis le XIX ${ }^{\mathrm{e}}$ siècle l'essor du roman en Russie, puis celui du cinéma en URSS. Le phénomène peut probablement expliquer l'importance des courants « réalistes » en Russie. 
feuilleton par le spectateur en soulignant la « vérité » de l'histoire par celle, indéfectible, du système axiologique induit par la structure actancielle du récit. Ainsi Hitler ou Bormann seront doublement mauvais, parce que l'Histoire telle que l'atteste les archives a montré leur folie sanguinaire, mais aussi parce qu'ils sont les «méchants » de la fiction, où ils remplissent, face à Stirlitz-le-héros, la valence d'opposants. La fictionnalisation des images d'archives simplifie l'histoire, la bipolarise en lui conférant la structure très nette du système axiologique du récit.

Les faits de la Deuxième Guerre mondiale tels qu'ils sont présentés par les archives servent de toile de fond fictionnelle au récit qui, lui, rejoue, à une échelle moindre - celle des scènes d'intérieur - une Deuxième Guerre mondiale réduite aux oppositions actancielles de tout énoncé narratif. Ainsi Hitler et Stalin apparaissent au début du feuilleton (Hitler dans le premier épisode, Stalin dans le second), non pas dans les images d'archives, mais comme actants de la fiction, et joués par des acteurs. Leur statut n'est toutefois pas exactement équivalent. Si Hitler est fictionnalisé (car représenté par un personnage interprété par un acteur), il apparaît dans une scène qui n'entretient pourtant pas de rapport avec le récit des aventures de Stirlitz (au point qu'on se demande pourquoi ce personnage est montré à l'écran : probablement, comme on l'a expliqué plus haut, pour baliser l'histoire grâce à la fiction). La scène se contente en effet de le montrer dans son bunker, donnant des ordres à ses subordonnés. Stalin, en revanche, apparaît dans une scène qui l'intègre à la fiction puisqu'il donne au chef du renseignement soviétique un ordre à transmettre à Stirlitz. Il y a alors risque de discrédit de la figure historique par le personnage fictionnel. Le risque est contourné par le travail sur le mythe. Alors qu'il donne des directives au chef des renseignements, Stalin prend une cigarette et en vide le tabac afin d'en bourrer sa pipe. Un champ/contrechamp montre les gestes de Stalin et le regard étonné et sentencieux de son subordonné. Il y a mise en scène du mythe dans le récit (et sur le visage du subordonné). En effet, l'histoire du tabac à cigarettes dans la pipe est l'une des nombreuses anecdotes historiques sur Staline, elle entretient le mythe du personnage. Faire figurer cette anecdote dans le récit permet d'y intégrer le mythe, qui authentifie - par référence à ce qui est largement connu - la fiction.

Ainsi le mythique permet d'authentifier la fiction pour la mettre en accord avec l'Histoire (mythique, c'est-à-dire déjà retravaillée) et de fictionnaliser l'histoire (dans le sens de la mettre en récit) afin qu'elle perde son statut d'énoncé à l'interprétation non codée, aléatoire. Les images montrant batailles de chars ou bombardements aériens deviennent alors les épisodes d'une sorte d'epos, dont le sens est unilatéral et la fin connue, comme est devinée, sinon connue, la fin envisageable de toute fiction et celle, mille fois répétée, du récit épique ${ }^{20}$.

20. Pour une analyse détaillée de l'emploi du mythique dans la fiction de télévision, cf. M. H. Goldsmith, «Video values education : Star trek as modern myth », Cultural Futures Research, 8,3,1984. 
Après cette étude de l'esthétique du feuilleton, je me propose de présenter quelques éléments d'analyse concernant le problème de sa réception, et, plus largement, du culte qui s'organise autour d'elle.

Comme je l'ai indiqué en introduction, la série reçut un bon accueil, et fut rediffusée à de nombreuses reprises entre 1973 et aujourd'hui. Ces rediffusions lui ont assuré un large public qui couvre une tranche d'âge de 20 (et probablement moins aujourd'hui, à l'heure où l'on édite le feuilleton en quatre cassettes vidéo) à 55 ans.

Si les réactions sont globalement positives, (je n'ai reçu d'écho défavorable de personne), l'interprétation du feuilleton varie selon l'âge et le sexe. Tous sont d'accord pour y voir un énoncé fortement idéologisé, avec une fonction très évidente de propagande, mais cela ne vient pas perturber leur réception positive $\mathrm{du}$ feuilleton. En effet, la propagande semble être un élément incontournable et admis, au point que les spectateurs interrogés n'y faisaient (font) guère attention. Invariant de tout énoncé à caractère public, l'idéologie uniforme n'attire guère l'attention, qui se reporte précisément sur l'élément instable d'un énoncé à l'autre.

Je retiendrai trois interprétations. La première est celle d'une Moscovite d'une cinquantaine d'années installée à Paris. La seconde celle d'une Moscovite d'une vingtaine d'année, la troisième celle d'un jeune homme d'une vingtaine d'années toujours, originaire d'Obninsk (région de Kaluga), mais installé à Moscou depuis quelques années. Les deux derniers sont étudiants.

Les trois personnes ont vivement rejeté l'interprétation des deux autres, élément qui tendrait à montrer la «personnalisation » (liée à son succès) de la série par les spectateurs $^{21}$.

La première interprétation est d'ordre herméneutique et, en l'occurrence, s'apparente au décodage idéologique. La personne interrogée y voit - en utilisant des catégories très littéraires participant de la culture de l'intelligentsia et/ou de la dissidence - un discours critique en langue d'Ésope sur la réalité soviétique des années 1970 : Stirlitz, à l'image des Russes, ou, en tout cas, de l'intelligentsia, dit (ou fait) une chose et en pense une autre.

Cette interprétation est très caractéristique d'un besoin de sens et de justification morale, souvent lié à une certaine tradition, idéaliste, forte en Russie. C'est probablement la concrétisation la plus proche - ce qui ne signifie pas la plus juste, cette notion n'ayant pas cours ici - de l'esprit dans lequel a été conçu le roman, puis, peut-être, la série, à moins que la série elle-même soit déjà le résultat d'un recul du thématique (bien qu'il y reste très fort, comme nous l'avons vu).

21. Sur ce phénomène de l'appropriation par l'interprétation des feuilletons télévisés, on consultera N. K. Baym, «Interpreting soap operas and creating community : Inside a computermediated fan culture », Journal of Folklore Research, 30, 2-3, 1993, p. 167 : « Soap operas are what Robert Allen [...] calls an "over-coded" form, meaning that they offer more possible interpretations than are necessary to move the narration forward. Because of this, the text can support multiple legitimate interpretations. », et p. $171:$ « [...] soap operas encourage multiple interpretations. » (C'est moi qui souligne). 
Les deux autres interprétations rejettent, à des degrés divers, cette importance accordée au message de l'œuvre. Elles empruntent des catégories appréciatives opposées à celle-ci, et très caractéristiques du contexte post-moderne22 .

La seconde, celle de l'étudiante, se pose en effet comme antinomique à la première. La jeune fille interrogée réagit vivement à l'interprétation herméneutique, et propose de voir dans Semnadcat' mgnovenij vesny un énoncé dominé par l'ironie. Il n'y a pas encore rejet du message, mais relativisation, mise en cause du sens, de l'interprétation unique. De plus, l'interprétation ironique - très valorisée en contexte post-moderne où elle constitue souvent la norme - permet, par un étrange mélange entre une certaine tradition russe de l'évaluation normative et le relativisme de l'appréciation selon la version vulgarisée du post-moderne, de récupérer la série. Cette deuxième concrétisation, de manière paradoxale, « sauve » (car il faut sauver) la série, en y voyant un énoncé post-moderne, alors qu'il semble bien, par son univocité idéologique, en être très éloigné. Elle marque en tout cas le moyen terme entre la première et la troisième interprétation, et est caractéristique du passage du feuilleton d'un contexte à un autre ${ }^{23}$.

La troisième interprétation est la plus caractéristique du contexte post-moderne. C'est celle de l'étudiant originaire d'Obninsk. Cette interprétation n'est pas unilatérale. En effet, je n'en retiens que le premier aspect, la façon dont l'étudiant m'a présenté le feuilleton alors que je ne l'avais pas encore visionné. Cette relecture totale de l'œuvre tendait à me présenter le feuilleton comme l'équivalent soviétique d'un quelconque feuilleton américain, avec sa thématique d'espionnage, son action et sa violence. Plus tard, l'étudiant modéra son propos en qualifiant par exemple le feuilleton de film (ce qui fait la différence avec les séries américaines), voire de film «d'auteur» (hudožestvennyj film)24. Mais ses premières descriptions, émaillées des histoires drôles que je présenterai plus loin, tendaient à augmenter l'action et à diminuer l'élément didactique du feuilleton.

Cette dernière interprétation évacue complètement le sens, le «message», si caractéristique de la première interprétation, et présente Semnadcat' mgnovenij

22. J'emploie évidemment le terme de «contexte post-moderne» et non «d'œuvre postmoderne », puisqu'il s'agit ici d'étudier la transformation radicale de la physionomie d'un énoncé du fait de sa survivance dans un contexte nouveau, marqué par l'arrivée de produits dissemblables mais apparentés par leur mode de diffusion (le prime time).

23. Il me semble que cette interprétation médiane, qui confirme son statut d'œuvre artistique à la série tout en l'adaptant au contexte nouveau en soulignant sa réflexivité, met en lumière le phénomène du glissement des œuvres entre diverses catégories et la difficulté d'une partie du public russe actuel à construire des catégories exclusives les unes des autres. $\mathrm{Cf}$., à ce sujet, S. Kuznecov, art. cit., p. 40 : «En s'avançant dans cette même direction [vers le cinéma à but ouvertement commercial], le cinéaste quitte inévitablement le territoire du soi-disant 'art' encore garant à Moscou de la possibilité de produire une œuvre culte » .

24. La traduction est problématique. Le plus souvent, la qualification hudožestvennyj film indique seulement qu'il s'agit d'une fiction, en opposition au documentaire. Dans le contexte de ma conversation avec l'étudiant, hudožestvennyj reprenait un peu de son sens premier d'« artistique ». 
vesny comme un « produit en apparence dépourvu des interrogations qui sont le lot commun des œuvres modernes, un produit de pur divertissement $»^{25}$.

À ce phénomène, caractéristique, à mon avis, de l'assimilation inconsciente par le plus grand nombre des catégories du contexte post-moderne en Russie postsoviétique ${ }^{26}$, s'ajoute celui d' « intellectualisation du kitsch » (l'expression est de Hannah Arendt ${ }^{27}$ ). En effet, l'étudiant, de même que de nombreuses autres personnes que j'ai interrogées, était conscient de la médiocre qualité de la réalisation (rythme trop lent, hyperinflation du discours, filmage grossier), mais légitimait son attachement au feuilleton - en réalité attachement à la culture de son enfance, voire à son enfance tout court - par une espèce de mise en scène auto-ironique d'une faute de goût assumée ${ }^{28}$.

Cette intellectualisation a besoin d'un discours légitimant. Celui-ci, censé légitimer le culte, a en réalité une double fonction. Il le légitime en même temps qu'il l'organise. Ce discours est, pour ce cas précis, essentiellement constitué des histoires drôles (anekdoty) consacrées aux aventures de Stirlitz.

Ainsi ces histoires drôles assument la fonction de rituel du culte, en même temps que de discours légitimant. Leur qualité pallie les défauts du feuilleton en le soumettant à un discours critique et en en proposant une relecture, en même temps qu'elles contribuent à entretenir la présence vivante du feuilleton dans la mémoire des téléspectateurs, ou permettent d'en conquérir de nouveaux (ce fut mon cas) ${ }^{29}$.

Ces histoires drôles sont d'ordres divers.

25. L. Jullier, L'écran post-moderne. Un cinéma de l'allusion et du feu d'artifice, Paris, L'Harmattan, 1997, p. 16.

26. Assimilation dont témoignent les médias russes « grand public », qui abusent du terme de «post-moderne », ainsi l'hebdomadaire moscovite Večernjaja Moskva, sorte de Nova mag ou Zurban, aujourd'hui disparu, titrait dans un numéro «Encore pire que le post-moderne » et, dans le numéro de l'été 1998, consacré aux Olympiades de la jeunesse, employait le terme « simulacre ». Dans des cercles plus restreints, artistiques ou intellectuels, le passage de la Russie au post-moderne est bien plus ancien. On consultera à ce propos l'article déjà cité de Leonid Heller, qui rappelle que l'introduction de la problématique post-moderne dans le travail de la critique russe émigrée date de la deuxième moitié des années 1980, et son introduction dans la vulgate soviétique de la fin de cette même décennie (art. cit., p. 5).

27. H. Arendt, « La crise de la culture. Sa portée sociale et politique », in La crise de la culture, Paris, Gallimard, 1972,p. 253.

28. Ce type de posture ironique est entré dans les modes de réception des œuvres en Russie, comme en témoigne la définition que donne S. Kuznecov d'un des types de motivations à l'origine de l'institution d'un culte pour un film. Cf. S. Kuznecov, art. cit., p. 40 : « L'exemple classique en sont les 'mauvais' films d'Ed Wood ou de Roger Corman qui sont mauvais au point qu'ils en deviennent bons. » (C'est moi qui souligne).

29. Cf. N. K. Baym, art. cit., p. 160 : «By making fun of the soap opera, r. a.t. s. [nom du site pris comme terrain d'étude pour l'enquête] participants, all of whom are soap opera fans, appropriate the weakenesses in the show and transform them into unique performances which themselves become a locus of group pleasure. This transformation helps keep the fans engaged, even when the soap opera fails to entertain on its own terms. Simultaneously, as the transformation takes on familiar forms over time, particuliar types of humor are traditionalized and serve salient functions in creating community. »

Sur le rôle actif des fans dans l'amélioration qualitative permanente du niveau de leur série préférée par le biais, entre autres, d'une relecture humoristique, on lira également H. Jenkins, Textual poachers : Television fans and participatory cultures, Londres, Routledge, 1992, p. 23 : 
Le premier groupe parodie des éléments du scénario original qu'elles reprennent en les inversant, les déplaçant, ou les décontextualisant. Comme celle-ci, portant sur l'élément de base du scénario, la situation de l'espion patriote :

«7-го ноября.

Штирлиц решил отпразновать. Надел пилотку красной армии, и пошел на работу. Приходит на работу, заходит к Мюллеру, и видит с удивлением, что Мюллер сидит в красной майке с надписью СССР, с серпом и молотом, и играет Подмосковные Вечера на баяне.

Штирлиц спрашивает: "В чем дело?"

Мюллер отвечает: “Да бросьте Штирлиц, неужели вас не мучит тоска по Родине?” »
« Le 7 novembre.

Stirlitz a décidé de le fêter. Il enfile une veste de pilote de l'Armée rouge, et va à son travail. Arrivé à son travail, il passe voir Müller et le découvre assis dans son fauteuil, en maillot rouge orné du sigle URSS, de la faucille et du marteau, jouant Podmoskovnye večera à l'accordéon.

Stirlitz, interloqué, demande : "Qu'est-ce que ça veut dire?"

Müller répond : "Ça va, Stirlitz, ne me dites pas que vous n'avez jamais le mal du pays." »

ou de celle-ci, caricaturant les invraisemblances du scénario, en en rendant les personnages « conscients » :

\begin{abstract}
« В бункере Гитлера проходит заседание. За большим столом сидят высшие офицеры Рейха, и обсуждают какие-то важные проблемы. Вдруг в комнату проходит Штирлиц. Подходит к сейфу, открывает его, берет там какие-то важные бумаги, и спокойно уходит.
\end{abstract}

Гитлер возмушается. Спрашивает : "Господа! Почему вы ничего не делаете? Кто это такое?”

Мюллер отвечает : “Аа, это Штирлиц, все равно выкрутится.” »
«Une réunion a lieu dans le bunker de Hitler. Assis autour d'une grande table, les hauts dignitaires du Reich débattent d'importantes questions. Entre Stirlitz. Il s'approche du coffrefort, l'ouvre, s'empare de documents importants et se dirige tranquillement vers la sortie.

Hitler, en émoi, demande: "Mais, messieurs, enfin, faites quelque chose. C'est qui, d'abord, celui-là?" Müller répond : "Bah, c'est Stirlitz, c'est pas la peine, de toute manière il s'en sortira." »

« Because popular narratives often fail to satisfy, fans must struggle with them... because the texts continue to fascinate, fans cannot dismiss them from their attention but rather must try to find ways to salvage them for their interests. Far from syncopathic, fans actively assert their mastery over the mass-produced texts which provide the raw materials for their own cultural productions and the basis for their social interactions. » 
ou de celle-là, déplaçant une formule célèbre employée par la voix off pour l'utiliser dans un contexte absurde :

«Штирлиц выходит из кафе, и падает лицом в лужу.

Голос за кадром: "Ровно через полчаса он встанет, и пойдет как ни в чем не бывало. Это была тоже одна из привычек, выработанная годами." »
«Stirlitz sort d'un café, et tombe le nez dans une flaque.

Voix off : "Dans exactement une demi-heure, Stirlitz se lèverait, et s'en irait comme si de rien n'était. C'était là aussi une de ces nombreuses habitudes, contractées avec les années." »30

Le deuxième groupe d'histoires drôles caricature en les dénudant les procédés stylistiques du récit et du filmage comme l'histoire suivante qui caricature l'emploi de la voix off 31 :

« Весна 1945-го года. В подвале Гитлера стоит очередь за краковской колбасой. Там все главы Рейха.

Как только открылось окошко Штирлиц нагло подошел, и сказал : "Мне два килограма."

Мюллер возмутился: “А что вы Штирлиц! Здесь все высшие офицеры Рейха."

Голос за кадром: "Мюллер не знал, что героям Советского Союза все полагается без очереди." »
«Printemps 1945. Dans les souterrains du bunker privé de Hitler on fait la queue pour un peu de mauvais saucisson. Dans la queue on trouve tous les hauts dignitaires du Reich.

À l'arrivée de la marchande, Stirlitz double tout le monde et demande : "Deux kilos s'il vous plaît."

Müller choqué, lui dit : "Ça va pas non, Stirlitz, qu'est-ce qui vous prend de doubler comme ça tous les hauts dignitaires du Reich."

Voix off : "Müller ne savait pas que les héros de l'Union Soviétique sont prioritaires dans les magasins.” »

Le troisième groupe d'histoires drôles caricature certains éléments extérieurs à l'univers pro-filmique, mais liés à l'histoire du feuilleton : ainsi celle-ci, qui se moque de l'auto-identification très forte de l'acteur principal, Vjačeslav Tihonov, à son personnage :

30. L'expression " Dans exactement une demi-heure, Stirlitz se lèverait, et s'en irait comme si de rien n'était. C'était là aussi une de ces nombreuses habitudes, contractées avec les années » est employée à la fin du 8 e épisode, quand Stirlitz décide de dormir un peu, avant de reprendre la route. La voix off explique alors que Stirlitz a appris à contrôler parfaitement la durée de son sommeil.

31. Cette dénudation des procédés stylistiques de la série par l'histoire drôle rappelle la pratique de l'explicitation comique des lois des soap operas par leurs fans, et remplit une fonction comparable. Cf. N. K. Baym, art. cit.,p. 161 : « Since these laws so often point out soap opera's absurdity, their articulation also helps members establish an explicit awareness of its absurdity that distances them from it and, indeed, makes that absurdity a source of humor in its own right. » 
« Штирлиц просыпается где-то в незнакомом месте. Белые стены. Окна нет. Болит голова. Заходят какие-то люди в белых халатах. Говорят : "Ну и напились вы вчера товарищ Тихонов.” »
«Stirlitz se réveille dans un endroit inconnu. Des murs blancs. Pas de fenêtre. Il a la migraine. Entrent des types en blouses blanches qui lui disent : "Qu'est-ce que vous vous êtes mis hier soir, camarade Tihonov." »

On voit se dessiner ici un élément de «culte»: la connaissance par le public d'éléments de l'histoire de la réalisation (du making of) du feuilleton, et la mise au même niveau (à travers la plaisanterie, même si le but de cette plaisanterie est précisément de moquer cette mise à niveau) d'éléments fictionnels et de faits réels.

Le dernier groupe d'histoires drôles témoigne enfin de la popularité des personnages du feuilleton, qui acquièrent dans les plaisanteries une véritable autonomie vis-à-vis de leur univers fictionnel d'origine : les personnages, essentiellement Stirlitz et Müller, deviennent prétextes à l'invention de jeux de mots dont le contenu est autonome et n'entretient aucun rapport de référentialité avec le scénario du feuilleton. Ainsi de ces deux histoires drôles, construites sur des jeux de mots ${ }^{32}$ :

« Штирлиц, говорит Мюллер, закройте, пожалуйста, окно. Дует.

Do it yourself, asshole, говорит Штирлиц . »

et :

« Штирлиц вошел в комнату, и выстрелил вслепую.

Слепая упала. »

Ces plaisanteries ont plusieurs fonctions. Inventées (probablement) d'abord pour opposer une résistance au discours de propagande du feuilleton, elles sont aussi la trace d'un attachement à quelque chose de familier. Elles éloignent donc en même temps qu'elles rapprochent. Elles amputent (d'une partie du sens) en même temps qu'elles procèdent à une relecture valorisante de l'œuvre et la popularisent. Elles sont l'expression principale du culte porté à Semnadcat' mgnovenij vesny.

Comme je l'ai précisé dans l'introduction, le phénomène du culte autour d'un feuilleton télévisé me paraît avant tout caractéristique de la société occidentale, puisqu'il s'organise autour d'objets du culte, produits dérivés d'une stratégie de marketing, le merchandising (ainsi peut-on trouver, dans certains magasins, en plus des fanzines et des disques, des T-shirts, des tasses à café, ou des figurines-jouets tirées de telle ou telle série américaine).

Ce type de stratégie était absent, à de rares exceptions près ${ }^{33}$, de la société soviétique et ne fait qu'apparaître aujourd'hui, ce dont peut témoigner la réédition

32. Ces deux dernières histoires drôles, fondées sur des jeux de mots propres au russe, ou à une paronymie bilingue, ne sont malheureusement pas traduisibles.

33. J'ai appris récemment de M. Pierre Doze, fan des aventures du héros de dessin animé Čeburaška, que l'on vendit à l'époque soviétique, entre autres produits dérivés, du dentifrice dans des tubes à l'effigie du héros inventé par E. Uspenskij. Si cette pratique révèle bel et bien l'existence d'un culte pour ce dessin animé, il ne me semble pas cependant qu'elle relève du 
récente en compact-disc de la musique (la bande originale, dirait-on en Occident) composée par Mihail Tariverdev pour Semnadcat' mgnovenij vesny ${ }^{34}$. En l'absence de ces produits dérivés - lieu d'investissement d'une participation personnelle - les histoires drôles remplirent (et remplissent encore) cette fonction. Ces anecdotes seraient autant de scenarii personnels dans lesquels le spectateur relocalise les personnages de la fiction, de même qu'en Occident, le jeu avec les figurines - pour les plus jeunes - ou la rédaction, par les fans de Star trek notamment, de récits prolongeant la série, serait l'expression de la "participation fictionnelle $»$ du spectateur ${ }^{35}$.

On peut dire que les histoires drôles remplissent de multiples fonctions à tous les stades de la réception du feuilleton télévisé en contextes soviétique et postsoviétique : elles soumettent le feuilleton à un filtre idéologique par leur fonction de mise à distance, elles opèrent ensuite une relecture de l'énoncé ainsi épuré, et le réinvestissent d'éléments inhérents à leur propre poétique (l'humour et l'animation contenus dans les histoires drôles sont absents du feuilleton lui-même, mais ce dernier profite d'eux tant ces histoires tendent à transformer le souvenir que l'on en a, ou l'idée que l'on s'en fait à l'avance). Elles transforment d'autre part sa perception - en contexte post-soviétique - conformément au nouvel horizon d'attente générique, façonné par l'importation en Russie des feuilletons télévisés américains (comme les plaisanteries présentant Stirlitz tirant au pistolet, ou sautant du toit d'un immeuble, motifs narratifs absents de la série). Notons que cette rencontre, ce conflit synchronique de deux éléments d'une diachronie, le feuilleton soviétique de 1973 et la série américaine des années 1980-1990 et l'appréciation de l'un en fonction du second, est un phénomène typiquement post-

merchandising, ne serait-ce que parce qu'elle ne pouvait avoir de but commercial dans le contexte d'une économie de type socialiste. Ainsi elle participe au culte de manière passive, en le référençant, et non active, en cherchant à l'étendre à des fins commerciales. Notons de plus que cette pratique fut assez limitée, et que la série Čeburaška a donné naissance, tout comme Semnadcat' mgnovenij vesny, à un nombre incalculable d'histoires drôles, véritable médium, à mon sens, du culte dont elle fait, elle aussi, l'objet. Sur le culte voué à cette série, on consultera P. Doze, « TCHEbourashka, la poupée russe qui se rue vers l'Ouest », in Citizen K International, XIII, « Luxe », hiver 1999/2000, p. 27.

34. On citera encore la publication, au début des années 1990, de livres dérivés de Semnadcat mgnovenij vesny. Toutefois, notons-le, ce sont les histoires drôles qui ont donné naissance à ce type d'ouvrages, qui se contentent souvent de rassembler ces histoires sous forme de recueils. Parfois cependant, il s'agit de véritables nouvelles, bon exemple de participation fictionnelle, mais là encore, leur esprit rappelle celui des histoires drôles, qui leur sont antérieures, et qu'elles viennent comme étirer. Voici les références, à titre d'exemple, du recueil que j'ai pu me procurer : B. Leont'ev, ed., Operacija « Šnaps » i drugie pohoždenija štandartenfjurera SS fon Štirlica (Opération «Schnaps » et autres aventures du Standartenführer SS von Stirlitz), Moscou, Izd.-vo MiK, 1992, coll. « Biblioteka parodii i jumora ».

35. Sur cette participation fictionnelle des fans de la série américaine Star trek, voir M. Jindra, «Star trek fandom as a religious phenomenon », Sociology of Religion, 55, 1, 1994, pp. $27-$ 51 , notamment p. 35 . Sur le marketing des produits dérivés, voir, dans le même article, la page 28 . 
moderne, amené par ce média éminemment post-moderne, par sa politique de diffusion, qu'est la télévision ${ }^{36}$.

La qualité du feuilleton est ainsi améliorée par l'éclat que lui confère l'image déformée qu'en renvoient les histoires drôles, et la permanence que semble lui assurer le succès de ces mêmes histoires (l'indépendance acquise par ces dernières vis-à-vis du feuilleton, telle que nous l'avons constatée plus haut, passe ici inaperçue). Elles excitent l'intérêt de l'ancien ou du futur spectateur, incité par elles à (re)voir le feuilleton. Elles servent d'objets du culte, de lieu de participation fictionnelle, et donc de réappropriation. Enfin, elles servent de code de reconnaissance, et sont à ce titre le signe d'un désir de (re)création d'une communauté.

Car c'est probablement ce phénomène plus global qui se cache derrière celui de l'organisation d'un culte pour les séries. Et ce désir est peut-être double. C'est le désir, tout d'abord, de se réapproprier un héritage culturel trop rapidement rejeté au moment de la déferlante de la culture populaire occidentale sur la Russie au début des années 1990. Ce rejet restant assez fort dans une partie de la population (jeune) - passionnée, non plus de culture occidentale tout court, comme il y a quelques années, mais de culture occidentale « underground »- il se crée un sousgroupe dans cette frange même de la population pour essayer de réhabiliter la culture de masse soviétique, en la transformant, par «intellectualisation du kitsch », en culture pop alternative à la culture pop occidentale.

Rejeter la culture soviétique paraît simpliste et revient à s'amputer d'une partie de son passé (souvenirs télévisuels liés à l'enfance), c'est-à-dire à se priver de la possibilité de refonder une communauté disparue d'une Russie contemporaine très marquée par les inégalités sociales et culturelles. Contre l'apparition d'une nouvelle forme d'inégalités, séparant les (jeunes) Russes ayant voyagé en Europe ou aux États-Unis des autres, ayant un accès ou non à la culture occidentale contemporaine (dont j'exclus les formes les plus grossières, diffusées dans tout le pays par les chaînes russes nationales), ce projet de recréation d'une communauté ${ }^{37}$ par la transformation en code culturel actif d'un système de références appartenant à une époque, l'URSS des années 1970-1980, où les inégalités dans l'accès à la culture n'existaient officiellement pas, illustre l'ambition de faire la synthèse entre culture russe soviétique et culture occidentale contemporaine (néo-camp), ambition en

36. Il me semble qu'un élément apparu après la rédaction du présent article peut venir confirmer cette analyse. Lors d'un séjour à Moscou, en juillet 1999, j'ai été frappé par une annonce diffusée tous les matins de semaine sur la première chaîne russe, aux alentours de 11 heures. L'annonce, qui signalait la rediffusion de la série à thématique guerrière Shvatka, bol'še čem žizn's'articulait autour du slogan suivant : « Le capitaine Kloss [héros de la série en question] a sa place entre Bond et Stirlitz». L'introduction, illégitime à première vue, ainsi que je l'ai expliqué plus haut, de Bond dans le paradigme sur lequel figure Stirlitz, ou, plutôt, l'introduction de Stirlitz dans le paradigme du film à la James Bond, participe d'une occidentalisation rétroactive du cinéma et de la culture télévisuelle russes soviétiques, facilitée par les raccourcis qu'opère la télévision de par sa politique de diffusion. Ce rapprochement Bond/Stirlitz confirme également la transformation de la réception du feuilleton lors du passage d'un contexte à l'autre.

37. Sur le rôle du culte pour les séries comme médium de création de communautés, cf. l'article, déjà amplement cité ici, de Nancy K. Baym. 
elle-même déjà post-moderne, et qui est le signe d'une réelle participation - audelà d'une logique d'influences réciproques - de la Russie au contexte culturel commun caractéristique de notre époque.

Septembre 1999.

Université de Rouen - Haute Normandie.

UFR des Lettres

Département de russe

76821 Mont-Saint-Aignan Cedex

e-mail :Rodolphebaudin@aol.com 